\title{
El recurso hídrico: ¿ético?
}

\section{The water resource: ethical?}

\author{
José S. Barba Nureña, $\unrhd$ Martín Bringas Maravi y Fabrizio Chirinos Delgado \\ Universidad Nacional Mayor de San Marcos. Lima, Perú.
}

Recibido: 27-11-2018 Revisado: 20-01-2019 Aceptado: 14-03-2019 Publicado: 25 - 05- 2019

\section{RESUMEN}

El impacto actual y proyectado del uso del recurso hídrico está fundado en los datos de numerosas investigaciones comprometidas con la sostenibilidad del medio ambiente y se enfoca la crisis actual que se vive por el buen manejo del recurso hídrico con que cuenta el planeta y que es de vital importancia para la vida y desarrollo de la especie humana, visto y analizado desde el punto de vista ético y de cómo se maneja este recurso, vital para la supervivencia no solo de la especie humana, ahora que ya se tomó conciencia de que conforme vayan pasando los años se va tornar más escaso afectando primordialmente en el desarrollo humano y la supervivencia del planeta.

Estas nuevas formas, generarán tendencias económicas pero que se usa muy bien un trasfondo ético que nunca estará mal quizás allí radique un enfoque más sistémico, no debemos llegar a dejar de actuar bien, sin tener que recurrir a alternativas que nos dan ansiedades que son condiciones para oportunistas.

Hoy, incluso con la mediación de acuerdos internacionales, ya se torna cada vez más difícil dar cumplimiento con las metas formuladas para retornar a condiciones tales que permitan la supervivencia del hombre y satisfacer con ello el principio de responsabilidad formulado por Hans Jonás: "Obra de tal modo que los efectos de tú acción sean compatibles con la permanencia de una vida humana auténtica en la tierra" (Rama, 2009).

Palabras clave: Ética ambiental, desarrollo sostenible, recurso hídrico.

\begin{abstract}
The current and projected impact of the use of water resources is based on data from numerous researches committed to the sustainability of the environment and focuses on the current crisis that is experienced by the good management of the hydric resource that the planet has and that is of vital importance for the life and development of the human species. seen and analyzed from the ethical point of view and how this resource is handled, vital for the survival not only of the human species.

As the years go by, it will become more scarce, affecting primarily human development and the survival of the planet.

These new forms will generate economic trends but an ethical background is very well used that will never be bad. If there lies a more systemic approach, we must not stop acting well, without having to resort to alternatives that give us anxieties that are conditions for opportunists.

Today, even with the mediation of international agreements, it has become increasingly difficult to comply with the goals formulated to return to such conditions that allow the survival of
\end{abstract}


man and satisfy with it the principle of responsibility formulated by Hans Jonás: "Obra de tal modo que los efectos de tú acción sean compatibles con la permanencia de una vida humana auténtica en la tierra" (Rama, 2009).

Keywords: Environmental ethics, sustainable development, water resources.

\section{INTRODUCCIÓN}

Muchas de las acciones emprendidas por el hombre son un "instrumento" para conseguir, a su vez, otro fin, otro bien.

Por ejemplo, nos alimentamos adecuadamente para gozar de salud, por lo que la correcta alimentación, que es un fin, es también un instrumento para conseguir otro fin: la salud.

¿Hay algún fin último? Es decir, ¿hay algún bien que se persiga por sí mismo, y no como instrumento para alcanzar otro bien? Aristóteles (2014) nos dice que la felicidad es el bien último al que aspiran todos los hombres por naturaleza. La naturaleza nos impele a buscar la felicidad, una felicidad que Aristóteles identifica con la buena vida, con una vida buena.

Pero no todos los hombres tienen la misma concepción de lo que es una vida buena, de la felicidad: para unos la felicidad consiste en el placer, para otros en las riquezas, para otros en los honores, etc.

No se trata de buscar una definición de felicidad al modo en que Platón busca la Idea de bien, toda vez que el intelectualismo platónico ha sido ya rechazado.

La ética no es, ni puede ser, una ciencia que dependa del conocimiento de la definición universal del bien, sino una reflexión práctica encaminada a la acción, por lo que ha de ser en la actividad humana en donde encontremos los elementos que nos permitan responder a esta pregunta.

Cada sustancia tiene una función propia que viene determinada por su naturaleza; actuar en contra de esa función equivale a actuar en contra de la propia naturaleza.

Una cama ha de servir para dormir, por ejemplo, y un cuchillo para cortar: si no cumplen su función diremos que son una "mala" cama o un "mal" cuchillo.

Si la cumplen, diremos que tienen la "virtud" (arete) que le es propia: permitir el descanso o cortar, respectivamente; y por lo tanto diremos que son una "buena" cama y un "buen" cuchillo. La virtud, pues, se identifica con cierta capacidad o excelencia propia de una sustancia, o de una actividad.

Del mismo modo, el hombre ha de tener una función propia: si actúa conforme a esa función será un "buen" hombre; en caso contrario será un "mal" hombre.

La felicidad consistirá por lo tanto en actuar en conformidad con la función propia del hombre. Y en la medida en que esa función se realice, podrá el hombre alcanzar la felicidad.

Si sus actos le conducen a realizar esa función, serán virtuosos; en el caso contrario, serán vicios que le alejarán de su propia naturaleza, de lo que en ella hay de 
característico o excelente y, con ello, de la felicidad.

En estos tiempos en los que nos desarrollamos, vivimos y vamos pasando la vida, podemos argumentar que el hombre vive implementando acuerdos, mecanismos, estrategias, programas, acciones y demás instrumentos ideados para lograr un desarrollo sostenible de lo que nos queda de la tierra, como planeta o como lugar donde habitamos.

Desde los inicios de la vida, el hombre ha sido el único beneficiado del ambiente y del agua, de forma que estos dos componentes de la tierra han atendido con holgura cada una de las necesidades del ser humano, inclusive abarcando deseos y placeres.

El evidenciar que el equilibrio se está rompiendo y que desfavorece la presencia de condiciones para la permanencia de la vida humana y no humana, se presentó el siglo pasado a finales de los años sesenta y comienzos de los setenta (Scotto J. et al., 1988; Meadows et al., 1992 y Miller et al., 2011).

Para esos tiempos, la ciencia disponía de la información necesaria para generar el conocimiento que describió como la actividad transformadora del hombre sobre el ambiente.

Se logró, así, alterar en dos milenios un escenario formado con el transcurrir de tiempos solo comparables con la escala geológica.

Fueron alteradas las condiciones del suelo, el agua y la atmosfera, cuyos efectos se relacionan directamente con problemas ambientales como el cambio climático glo- bal, la destrucción de la capa de ozono, la pérdida de diversidad, la contaminación de los océanos, la escasez y mal uso del agua, la perdida y degradación de suelos agrícolas y forestales, la desertificación, la lluvia acida, entre otros.

El simple hecho de que todos estos fenómenos sucedan amenaza la existencia de la vida en la tierra, dando sentido a la cita de Leakey y Lewin en 1997: "Dominante como ninguna otra especie en la historia de la vida en la tierra, el homo sapiens está a punto de causar una gran crisis biológica, una extinción en masa, el sexto acontecimiento de estas características que habrá ocurrido en los últimos 500 millones de años".

Conscientes de lo que puede pasar en el futuro, al agua hay que valorarla como un recurso natural indispensable para la vida, cuyo consumo aumenta de manera muy acelerada, y si bien el recurso agua podría considerarse como renovable, su calidad disminuye de manera paulatina lo que puede dar lugar a problemas por escasez.

Además, y aunque tres cuartas partes del planeta están cubiertas de agua, sólo una mínima parte es apta para el consumo humano.

\section{EL AGUA Y EL SER HUMANO}

La importancia del agua para la vida en la tierra se debe principalmente a que es el componente mayoritario en la estructura de los seres vivos, esencial para su metabolismo.

Es el vehículo de transporte delos elementos nutritivos, es básico para mantener la turgencia de las células y participa en todos los procesos y reacciones químicas. 
Por otra parte, regula la temperatura ambiental del planeta y es un factor social que ha condicionado el desarrollo de las diferentes civilizaciones, siendo actualmente un recurso económico fundamental.

Está estimado en la actualidad un consumo diario medio de agua por persona de unos 200 litros, incluyendo importantes pérdidas de distribución.

El agua procede de embalses y pozos, desde donde es transportada a través de grandes conducciones, potabilizada en una estación de tratamiento y, a continuación, elevada a depósitos.

Este proceso de producción y transporte, en última instancia del agua de lluvia, con lo que en épocas de sequía se corre el riesgo de sufrir restricciones.

La mayor parte del agua que entra en nuestras casas sale de forma residual cargada de diversos contaminantes que van camino de las depuradoras.

Estas están diseñadas únicamente para neutralizar los contaminantes orgánicos, nunca los compuestos tóxicos que se acostumbran a tirar por lo que el proceso de depuración pierde capacidad.

Dado que en la zona donde vivimos el agua disponible varía mucho dependiendo de la climatología, el ahorro debe ser considerado como una premisa fundamental y se convierte en factor de garantía de buena calidad, independientemente de la disponibilidad puntual del recurso.

La utilización racional del agua impone una gestión eficiente y sostenible del recurso y nos compromete a ser cuidadosos con el gasto del agua, tanto a nivel individual como colectivo.

Estos recursos serán de utilidad para atender aumentos estacionales del consumo de agua.

Entendemos como uso sostenible del agua el propósito de hacer compatible el uso de un bien natural con el mantenimiento del ecosistema al que pertenece y además que no se produzca una pérdida de las funciones para satisfacer la demanda.

Así, se evitan situaciones de sobreexplotación. La gestión sostenible del agua consiste en administrar los recursos hídricos, tanto subterráneos como superficiales, en base a un correcto balance entre las necesidades de la sociedad y el medio ambiente natural.

\section{EL AGUA: DESARROLLO ECONÓMICO Y SOCIAL}

El agua no es sólo un elemento imprescindible para la vida, sino también un medio de desarrollo económico y social.

Al mismo tiempo, es un instrumento que condiciona la organización del territorio al conformar un sistema básico en la construcción de un espacio cohesionado e integrado que determina la capacidad de desarrollo y las condiciones de vida de la población (Opaluch, 1982).

En nuestros días, es unánime la opinión de que el agua no es un recurso ilimitado que garantice indefinidamente el suministro para el abastecimiento urbano y la producción de alimentos o energía.

Considerar lo contrario lleva inevitablemente a la degradación de los ríos, la sobreexplotación de los acuíferos y la desertización del territorio (Raggi, et al., 2013). 
La consideración del agua como un recurso limitado implica la necesidad de incorporar criterios de eficiencia y sostenibilidad en su gestión.

El coste real del agua es superior a los costes de suministro y depuración, de lo cual se deduce la importancia de realizar prácticas que reduzcan el consumo (Nauges y Thomas, 2000; Saleth y Dinar, 2000).

En las últimas décadas, se ha generado una preocupación internacional creciente por la escasez de este recurso en determinadas zonas del planeta, ya que el incremento de la población tiene un efecto directo sobre su demanda, y cada vez cuesta más hacerla llegar donde se necesita.

La escasez de agua implica una menor producción de alimentos y un freno en el desarrollo industrial, factores que serían detonadores de una crisis mundial cuyas consecuencias afectarían, sobre todo, a los países en desarrollo.

Es en la década de 1990 cuando numerosos expertos comienzan a dar la alarma acerca del inadecuado manejo de los limitados recursos hídricos, despertando la conciencia de los diferentes sectores involucrados, pese a que ya había estudios y frases de entendidos en el tema que referencian estos a décadas de los 70 .

La crisis mundial del agua es uno de los problemas clave que condicionan el desarrollo sostenible. La escasez del recurso, que se verá agravada por los efectos del cambio climático, su irregular distribución geográfica y temporal, y la creciente demanda de los distintos usos son los elementos que configuran esta crisis mundial.
Tanto la Cumbre del Milenio como la última Cumbre de la Tierra han asumido, entre sus objetivos y compromisos más relevantes, reducir a la mitad la población que no tiene acceso a agua potable para el 2020.

Aunque el problema afecta más intensamente a los países en desarrollo (más del 50 \% de esta población está expuesta a fuentes de agua contaminadas), las tensiones entre los distintos usos del agua en competencia, así como entre los distintos países o regiones que comparten el recurso, se producen constantemente en distintos territorios del planeta.

\section{LA ÉTICA PARA ENFRENTAR EL PROBLEMA DE LA ESCASEZ DEL RECURSO HÍDRICO}

En el análisis que merece el problema de la escasez o que desaparezca el recurso hídrico, (ONU, 2006) define: "...ética en sinónimo de la moral. Sin embargo, el concepto de la ética alude a enfoques filosóficos, mientras que el termino moral tiene en cuenta aspectos sociales y religiosos..."

Se puede aceptar que la ética es la filosofía de la moral y los valores morales son un producto cultural del hombre, por lo que estos se construyen en función de la relación que el hombre ha logrado con el ambiente en busca del bien común (Arrojo, 2006).

La composición variada que exhiben los recursos naturales disponibles en el ambiente, para diferentes sitios geográficos de un mismo territorio, obligan a la concepción de una moral particular que refleja la realidad a la cual el hombre se pretende adaptar.

Los valores morales del mundo occidental nos han llevado directamente a una crisis que toca lo ambiental, lo social, lo político y lo económico, lo cual exige soluciones que 
permitan el desarrollo sostenible de la vida humana y no humana en la Tierra.
En la figura 1 se muestra la comparación entre ambas posiciones.

\begin{tabular}{|c|c|}
\hline Ecología superficial & Ecología profunda \\
\hline $\begin{array}{l}\text { La diversidad natural es valiosa } \\
\text { como un recurso para nosotros. }\end{array}$ & $\begin{array}{l}\text { La diversidad natural tiene su } \\
\text { propio valor intrínseco. }\end{array}$ \\
\hline $\begin{array}{l}\text { No tiene sentido hablar acerca del } \\
\text { valor, excepto como valor para la } \\
\text { humanidad. }\end{array}$ & $\begin{array}{l}\text { Igualar valor con valor para } \\
\text { humanos revela un prejuicio racial. }\end{array}$ \\
\hline $\begin{array}{l}\text { Las especies de plantas deberian } \\
\text { ser salvadas debido a su valor como } \\
\text { reserva genética. }\end{array}$ & $\begin{array}{l}\text { Las especies de plantas deberian } \\
\text { ser salvadas debido a su valor intrínseco. }\end{array}$ \\
\hline $\begin{array}{l}\text { Recursos significa recursos para } \\
\text { los humanos. }\end{array}$ & $\begin{array}{l}\text { Recursos significa recursos para } \\
\text { los seres vivos. }\end{array}$ \\
\hline $\begin{array}{l}\text { La naturaleza es necesariamente } \\
\text { cruel. }\end{array}$ & $\begin{array}{l}\text { El hombre es cruel pero no } \\
\text { necesariamente. }\end{array}$ \\
\hline
\end{tabular}

Figura 1. Paralelo entre ecología superficial y profunda. (Delgado, 2005)

Se nota la tendencia utilitarista de la ecología superficial, ya que identifica a las demás especies como una fuente potencial de beneficio para el hombre, mientras que la ecología profunda implica la expansión de la ética más allá del dominio humano al considerar criterios no utilitaristas construidos sobre bases teológicas, evolutivas, deontológicas, entre otras.

Estos criterios contienen elementos que intentan unificar al hombre con los demás seres vivos y esto, desde el punto de vista filosófico, ubica a la ecología profunda en una posición monista denominada por algunos como eco humanismo, lo que significa una clara contraposición al antropocentrismo que ha cultivado el mundo occidental.

El futuro de la humanidad depende de los acuerdos que se logren con respecto al uso de sus recursos naturales, siendo necesario un cambio ético de actitud en el comportamiento individual (Trelease, 1974).

La construcción de la cultura humana ha girado en torno de los recursos naturales y entre ellos el agua cobra la mayor importancia, ya que se diferencia de los otros por su maravillosa propiedad de renovarse continuamente a causa del ciclo hidrológico (D.R.N.A., 2008) El agua ha sido, desde siempre, el fundamento de la vida.

Leonardo da Vinci la llamaba "Sangre de la naturaleza".

El origen de todas las cosas lo veía Thales de Mileto en el agua, en tanto que Empédocles situaba las cuatro esquinas de los elementos en "la tierra, el aire, el fuego y el agua", al tiempo que la filosofía anterior a Sócrates reconstruye los cuatro elementos de la prosperidad común en "tierras, aguas, hombres y dinero". 
El valor simbólico del agua entre dichos recursos básicos es hoy día tan importante para el medio ambiente como lo era en aquellos tiempos.

Aunque el Principio No. 4 de la Declaración de Dublín sobre el Agua y el Desarrollo Sostenible le agrega un valor económico, en realidad el agua "también tiene valores intangibles, simbólicos, culturales, religiosos, que hacen, prácticamente imposible que se pueda tratar como un bien de consumo usual" (Llamas, 2008).

Dada la diferencia en la concepción del valor del agua, el hombre se ha visto en la necesidad de resolver conflictos, atenuar riesgos y ofrecer equidad ante situaciones negativas derivadas del uso del agua, afirma que existen implicaciones éticas en muchas de las facetas de la gestión del agua y que dichos aspectos éticos giran en torno a cuestiones como: "¿Quiénes participan y en qué tipo de decisiones intervienen? ¿Tienen la posibilidad de formular alternativas, o sólo de reaccionar ante las soluciones ya formuladas por otros? ¿Cuál es la base para valorar, implícita - explícitamente, las compensaciones económicas? ¿Qué tipo de información debe ser accesible al gran público y cómo se facilita el acceso? ¿Cómo se caracterizan los impactos ambientales y cómo son incluidos en el proceso? ¿De qué modo intervienen los expertos y los no expertos? ¿Qué uso o abuso se hace de la información científica o técnica de los expertos?" (Llamas, 2008).

La gestión y manejo del agua ha evolucionado conforme se adquiere mejor conocimiento de las interdependencias que se dan entre los elementos que están involucrados como son ambiente, política, economía y sociedad. Inicialmente se consideró una estrategia de mandato y control soportada por la aplicación de instrumentos derivados de leyes, estándares, reglamentos, códigos, etc.

Pero no fue suficiente y se dio paso a la formulación de instrumentos económicos que involucraron la creación de impuestos, tarifas y subsidios.

En esta estrategia, no se aseguró equidad y fue entonces necesario asumir una estrategia consensual, fundamentada en la ejecución de lo dispuesto por audiencias donde tuviesen oportunidad de participar todos los afectados.

Hoy, aunque todos estos elementos se conjugan en cada una de las etapas de la gestión del agua, no resultan suficientes, puesto que los conflictos, las necesidades y los problemas surgen más rápido que las soluciones.

Pickering (1996) propone que una forma de responder a esta situación es "apelar a los valores éticos de las personas (y desarrollar en ellas algunos nuevos valores), como medida para contrarrestar esos retrasos... Desarrollar valores éticos, tanto en los consumidores, como en las autoridades y en la industria, tiene un potencial mayor, más efectivo y más económico que la regulación compleja del agua, además de que sería mucho más aceptable".

Actualmente, se estima que los criterios de análisis que se deben considerar en la Gestión Integral del Recurso Hídrico son (Solanes y Getches, 1998):

\section{- Eficiencia}

Los sistemas de asignación de agua pueden ser usados de varias maneras para garantizar un uso eficiente del recurso.

Una de éstas es el reconocimiento del valor económico del agua. 
Los mercados pueden distribuir agua entre consumidores consistente con el valor que la sociedad ha asignado al recurso.

La asignación de un bajo valor al uso del agua resulta en usos ineficientes, con una tendencia a no dejar agua para otros usos, con agotamiento de las fuentes y el consecuente daño de los ecosistemas.

\section{- Equidad}

Históricamente, el derecho al uso de agua ha sido condicionado a obligaciones recíprocas entre el usuario y la comunidad.

En general, la definición de "lo justo" depende de la concepción de la comunidad.

Por lo tanto, los métodos usados para tomar decisiones y racionar el agua deben involucrar los intereses comunitarios, fundamentados en información adecuada y no en opiniones arbitrarias.

\section{- Sostenibilidad ecológica}

Los conceptos de equidad y eficiencia se centran en asignar agua a necesidades humanas fundamentales.

Sin embargo, los valores ecológicos no están dirigidos exclusivamente a necesidades humanas.

Los ejemplos más ilustrativos del impacto de las decisiones basadas primariamente en motivos ecológicos lo constituyen los relacionados con la conservación de especies en peligro.

- Equilibrio

El desarrollo de recursos para satisfacer las necesidades actuales de la población, mientras se conservan recursos suficientes para beneficio de las generaciones futuras, es un propósito muy aceptado en el discurso internacional sobre la gestión de recursos naturales.

El concepto de equilibrio no sugiere una fórmula exacta para asignar valores a intereses distintos.

Tampoco significa que todos los intereses se estiman de una manera igual.

El ideal de equilibrio sugiere que se deben considerar todas las consecuencias en el proceso de la toma de decisiones.

Ningún interés debe ser excluido del proceso y ninguno debe tener una influencia desproporcionada al valor que la sociedad le asigna.

Hacer operativos estos criterios implica la revisión de cuestiones éticas entre las organizaciones que buscan articularlos, de esa manera se observarán las mejores formas de preservar el líquido elemento, necesario para la subsistencia de la especie humana, así como del planeta mismo.

\section{EL CONSUMO DEL AGUA, ¿TIENE CONTROL O ES EXCESIVO?}

El agua es un recurso escaso. Cuando el usuario percibe un costo bajo tiende a consumir más agua y con ello se genera escasez, por lo que esta no es evidencia de falta de recurso.

Por el contrario, se constituye en evidencia de un exceso de demanda.

El usuario debe ser consciente de que el recurso agua es limitado y su demanda se debe controlar; de lo contrario, a largo plazo no podrá garantizarse un suministro adecuado. 
Surge entonces la tarifa como mecanismo para controlar la demanda, aunque esta intención se puede ver afectada por el subsidio que reciben los estratos bajos.

Así, crear una cultura de ahorro de agua es un reto bastante complejo.

Pensemos que todo usuario se relaciona con la empresa prestadora, a través de un contrato de prestación de servicios, que lo obliga a pagar lo consumido y el costo solo se verá reducido en la medida que consuma menos; si se pasa de un tope se le cobrará más (Rodríguez Martín, 2003).

Se obliga al ahorro de agua a través de un instrumento económico, en lugar de construir conciencia del problema que se vive: la escasez del recurso hídrico.

Si se genera conciencia, se construye un nuevo esquema de pensamiento y con ello una nueva cultura, cambia el valor moral del consumidor y este entenderá que si el ahorro de agua no comienza a partir de un cambio en su actitud, no será posible garantizar el recurso para atender las necesidades de las futuras generaciones.

También es necesario pensar que el agua es de todos, de esta forma, la sociedad debe estar involucrada en la toma de decisiones respecto de la gestión del recurso hídrico. Se logra mayor claridad en los aportes de la sociedad cuando esta se encuentra debidamente informada de la problemática local.

El diálogo constructivo entre todos los actores involucrados es provechoso en la medida en que converjan la sabiduría popular, el conocimiento técnico, el modelo económico y la intención política.
De esta forma, se logra incluir, en la gestión del recurso hídrico, elementos de valor moral como la equidad, la solidaridad y la responsabilidad.

Al considerar la sociedad, se pueden tomar las decisiones en el nivel social más bajo, atendiendo el criterio de subsidiariedad: un principio ético importante en el sentido de que un órgano superior no decida lo que puede ser competencia adecuada de otros organismos inferiores más próximos al usuario y de menor ámbito territorial.

\section{EL ESTADO PERUANO Y SU RESPONSABILIDAD DE UNA POLÍTICA DE PRESERVACIÓN DEL RECURSO HÍDRICO}

En este sector recae la responsabilidad de generar una política de desarrollo sostenible, es decir, un esquema que permita atender nuestra necesidad sin poner en riesgo las futuras generaciones.

Entonces, surge una cuestión ética que pocas veces es considerada: ¿con qué derecho nos atrevemos a establecer las necesidades de las futuras generaciones? Al generar un escenario se está condicionando la libertad de desarrollo que debe existir en nuestra descendencia.

Hay una obligación ética de entregar a nuestros sucesores un entorno en iguales o mejores condiciones de las que lo recibimos de nuestros predecesores.

Es obligación de las instituciones reducir la incertidumbre en las proyecciones y mejorar con ello los escenarios en los que se proyecta la gestión del recurso hídrico (Eto, 2008).

El aprovechamiento sectorial del recurso hídrico no se da en un ambiente en el que la 
información se comparta plenamente.

La sociedad, en su conjunto, debe exigir acceso a la información dado que ésta influye en los procesos de decisión.

La mayoría de estas instituciones son de naturaleza pública y es difícil acceder a la información, incluso se debe pagar por ella cuando se supone que debería estar disponible al público.

Existiendo tecnología disponible para la gestión de información, las instituciones deben considerar un sustancial mejoramiento en la adquisición y transmisión de datos a toda persona interesada (C.I.U.P., 2011).

Es usual la falta de recursos económicos en estas instituciones del Estado. Les resta capacidad de planificación y de gestión.

La carencia de recursos económicos es referida continuamente como un impedimento para lograr un mejor desempeño. Sin embargo, estas entidades disponen de presupuestos atractivos que las constituyen en un verdadero fortín para la burocracia que manejan los políticos y lamentablemente no dejan de estar involucradas en escándalos asociados con manejos indecorosos, no escapándose de la corrupción que envuelve a los diferentes niveles del gobierno peruano: nacional, regional y local.

\section{CONCLUSIONES}

- Las cuestiones de justicia, ética y moralidad son totalmente subjetivas, controvertidas y difíciles de exponer.

- Dependen de los valores y aspiraciones de cada ser individual. La intención de este artículo no es tanto convencer sobre una posición definitiva, sino exponer una serie de ideas, inquietudes, frases y sugerencias. Toca a quien escuche o lea estas ideas, meditar en ellas, valorar si son correctas, y considerar como podrían implementarse si fuese el caso.

- La ética y la moralidad debieran entenderse como actitudes, maneras de ser y de actuar, individuales. Es decir, no se puede ni debe forzar la moralidad y la ética en otros, salvo mediante el ejemplo. Cuando hay consenso de varios sobre algún estilo de comportamiento que debe generalizarse, entonces este se implantará mediante las leyes y la legislación, aunque eso normalmente es menos profundo y más lento que la verdadera ética.

- Como parte de la solución se ha planteado la necesidad de construir nuevos valores, los cuales deben ser producto de la reconciliación del hombre con la naturaleza. Planteamientos teóricos aportados por la ecología superficial y la ecología profunda nos permiten reflexionar respecto del cambio de actitud que debemos afrontar, para hacer frente a los diferentes problemas ecológicos que se vienen presentando con el recurso hídrico.

- Es ético tener transparencia en la información ante la ciudadanía. No limitarse a lo mínimo que exigen algunas leyes, normas, convenios. Desarrollar la disciplina a nivel de gobierno y ciudadanía, para que sistemáticamente se generen y difundan informes completos, entre el personal interno y hacia el usuario.

Un aspecto ético, deseable para una organización, es hacer entender a los encargados de la administración del recurso hídrico en favor de las mayorías, está muy por encima de su puesto y estatus, así que debe tener la humildad de no aprovechar su cargo para buscar posiciones de status personal, o de 
sentirse autónomo y poderoso, cuando es evidente que los problemas urbanos, sociales y ambientales demandan enfoques integrales, y planteamientos interdisciplinarios.

\section{REFERENCIAS BIBLIOGRÁFICAS}

Agua, I. M. (s.f.). Centro Virtual de Información del Agua. México: Fondo para la Comunicación y la Educación Ambiental.

Aristóteles. (2014). Ética a Nicómaco. Wikisource.

Arrojo, P. (2006). El reto ético de la nueva cultura del agua: Funciones, valores y derechos en juego. Barcelona: Ediciones Paidós Ibérica, S.A.

Centro de Investigación de la Universidad del Pacífico - CIUR. (2011). Estimación de los beneficios económicos y sociales del Proyecto de Irrigación Olmos. Lima.

Conferencia Internacional sobre el Agua y el Medio Ambiente (1994). Water Markets and the Principles of Dublin. Trabajo presentado en la conferencia de la Asociación Mundial para el Agua, 4-8 de noviembre, Windhoek, Namibia.

Delgado, C. (2005). Filosofía de la ciencia y bioética. Revista Cubana de Filosofía. Edición Digital. Nro 2.

Departamento de Recursos Naturales y Ambientales. (2008). Plan integral de recursos de agua. San Juan, Puerto Rico: Estado Libre Asociado de Puerto Rico.

Eto, G. (2008). El desarrollo del Derecho Procesal Constitucional a partir de la Jurisprudencia del Tribunal Constitucional Peruano. Centro de Estudios Constitucionales.
Editorial Gráfica CARVIL. S.A.C. Lima.

Garcia, P. y Obregón, N. (2012).

Consideraciones de ética ambiental en la Gestión Integral del Recurso Hídrico. Cali, Colombia: Entramado.

Lewin, R. y Leakey, R. (1997) El futuro de la vida y de la humanidad. Editorial TUSQUETS EDITORES ISBN: 9788483105511

Meadows, D. H., Meadows, D. L. y Randers, J. (1992). Beyond the Limits. Post Mills, Vermont: Chelsea Green Publishing Company.

Miller, L., Gans, F. y Kleidon, A. (2011). Estimating maximum global land surface wind power extractability and associated climatic consequences. Earth Syst. Dynam., 2, 1-12.

Nauges, C. y Thomas, A. (2000). Privatelyoperated water utilities, municipal price negotiation and estimation of residential water demand: The case of France. Land Economics, 76, pp. 6885.

PNUD (2006). Informe sobre desarrollo humano. Madrid: Grupo Mundi Prensa.

Opaluch, J. (1982). Urban residential demand for water in the United States: Further discussion. Land Economics, 58 (2), pp. 224227.

Organización de las Naciones Unidas. (2006). Escasez de agua, riesgo y vulnerabilidad. Más allá de la escasez: Poder, pobreza y la crisis mundial del agua. Informe sobre Desarrollo Humano. Programa de las Naciones Unidas para el Desarrollo, 133-170.

Phillips, Ch. (1993). The Regulation of Public Utilities. Estados Unidos: PUR, pág. 838. 
Pickering, G. (1996). Necesidad de una Política Nacional de Aguas. En Seminario Internacional

Raggi, M., Sardonini, L. y Viaggi, D. (2013). The effects of the Common Agricultural Policy on exit strategies and land re-allocation . Land Use Policy, 31, 114-125.

Rama, C. (2009). Encuentro de Educación a Distancia de la IESALC UNESCO, Puebla.

Rodríguez Martin, J. (2003). El derecho ciudadano al agua potable. Revista Jurídica de la Universidad Interamericana de Puerto Rico, 37 (2), 477-491.

Saleth, R. y Dinar, A. (2000). Urban thirst: Water supply augmentation and pricing policy in Hyderabad City, India. Tecnical Paper 395, The World Bank, Washington D.C.

Scotto, J., Cotton, G. y Urback, F. (1988). Biologically Effective Ultraviolet Radiation: Surface Measurements in the United States, 1974-1985. Science Vol. 239,762-764.

Singer, P. (s.f.). Ética para vivir mejor. Ariel. Solanes, M. y Getches, D. (1998) Prácticas recomendables para la elaboración de leyes y regulaciones relacionadas con el recurso hídrico. Washington, D.C. No. ENV-127.

Trelease, F. (1974). Water Law, Resource Use and Environmental Protection. West Publishing Company, St. Paul Minnesota, Estados Unidos, 147. 